\title{
Artificial Intelligence in the healthcare of older people
}

\section{Elizabeta B Mukaetova-Ladinska ${ }^{1,2 *}$, Tracy Harwood ${ }^{3}$ and John Maltby ${ }^{1}$}

${ }^{1}$ Department of Neuroscience, Psychology and Behavior, University of Leicester, Leicester, UK ${ }^{2}$ The Evington Centre, Leicester General Hospital, Leicestershire Partnership NHS Trust, Leicester, UK ${ }^{3}$ De Montfort University, The Gateway, Leicester, UK

\section{Abstract}

Clinical applications of Artificial Intelligence (AI) in healthcare are relatively rare. The high expectations in relation to data analysis influencing general healthcare have not materialized, with few exceptions, and then predominantly in the field of rare diseases, oncology and pathology, and interpretation of laboratory results. While electronic health records, introduced over the last decade or so in the UK have increased access to medical and treatment histories of patients, diagnoses, medications, treatment plans, immunization dates, allergies, radiology images, laboratory and test results, these have potential for evidence-based tools that providers can use to make decisions about a patient's care, as well as streamline workflow. In the following text, we review the advances achieved using machine learning and deep learning technology, as well as robot use and telemedicine in the healthcare of older people.

\section{Key points:}

1. Artificial Intelligence use is extensively explored in prevention, diagnosis, novel drug designs and after-care.

2. Al studies on older adults include a small number of patients and lack reproducibility needed for their wider clinical use in different clinical settings and larger populations.

3. Telemedicine and robot assisted technology are well received by older service users.

4. Ethical concerns need to be resolved prior to wider Al use in routine clinical setting.

\section{More Information}

*Address for Correspondence: Elizabeta B Mukaetova-Ladinska, Department of Neuroscience, Psychology and Behaviour, University of Leicester, Leicester LE1 7RH, UK, Email: eml12@le.ac.uk

Submitted: 20 February 2020

Approved: 19 March 2020

Published: 20 March 2020

How to cite this article: Mukaetova-Ladinska EB, Harwood T, Maltby J. Artificial Intelligence in the healthcare of older people. Arch Psychiatr Ment Health. 2020; 4: 007-013.

DOI: 10.29328/journal.apmh.1001011

Copyright: @ 2020 Mukaetova-Ladinska EB, et al. This is an open access article distributed under the Creative Commons Attribution License, which permits unrestricted use, distribution, and reproduction in any medium, provided the original work is properly cited.

Keywords: Artificial intelligence; Dementia; Geriatric Medicine; Older People; Healthcare

\section{Check for updates}

OPEN ACCESS

\section{Introduction}

Artificial Intelligence (AI) has infiltrated our social lives and expanded to AI-based services and tasks, including areas with a large impact on people, such as healthcare. The shortages of staff working in the UK health and social system requires alternative arrangements to meet the everincreasing health needs, especially of the ageing population, in terms of both streamlining the current health resources and making them more efficient. AI use is extensively explored in prevention, diagnosis, novel drug designs and after-care, and thus, may drive meaningful changes across the entire patient journey (Table 1), but remains still restricted mainly to cancer, neurological (i.e. stroke, Parkinson's disease) and cardiovascular diseases [1].

Although people from all ages benefit from the above technological advances, different age groups may have distinct health care needs. Thus, in contrast to younger adults, whose priorities are inpatient experiences [2], older people seem to face a number of barriers in contacting communitybased primary health care, such as limited access, lack of standardized information systems and care pathways [3], all necessary to address their complex health care and social care needs. Indeed, older adults have much higher prevalence of nearly all major chronic and long-term conditions. In addition, they are more likely to succumb to adverse health events, such as a falls or infections, and these can lead to dramatic changes in their physical and mental wellbeing even after an apparently minor incident [4]. However, person and familyfocused care, self-management resources, and successful collaborative practice have been all highlighted as facilitators of good health care provision both by older people and their families [3]. All the above places the importance of diagnosis, monitoring of disease risks and their prevention, as well as management and optimizing of geriatric syndromes in the community for both older people living independenly or in $24 \mathrm{~h}$ care facilities. In particular, identification of health issues/ diagnosis, support/treatment needs evaluation, development 


\begin{tabular}{|c|}
\hline $\begin{array}{l}\text { Medical records } \\
\text { Data mining of medical records to provide better and faster health services } \\
\text { Reducing time on administration and make healthcare more patient-centric }\end{array}$ \\
\hline $\begin{array}{l}\text { Prediction and diagnosis of diseases: } \\
\text { Extracting phenotypic features and clinical laboratory results from case reports to enhance diagnostic accuracy } \\
\text { Diagnostic imaging } \\
\text { Genetic screening } \\
\text { Electrodiagnosis (i.e. electromyelography) } \\
\text { Disability evaluation } \\
\text { Mass screening } \\
\text { Disease monitoring }\end{array}$ \\
\hline $\begin{array}{l}\text { Drug development and improvement in therapeutic use } \\
\text { Identifying disease targets for therapeutic intervention } \\
\text { Identifying drug candidates; repositioning of available drugs } \\
\text { Identifying the best drug combinations for each patients' health requirements } \\
\text { Gene editing } \\
\text { Replacing animal testing and detecting toxicity of chemicals/agents } \\
\text { Speeding up clinical trials } \\
\text { Identifying biomarkers to diagnose disease }\end{array}$ \\
\hline $\begin{array}{l}\text { Personalized treatment plan (ready to be implemented in clinics) } \\
\text { ctDNA: circulating tumour DNA testing for treatment selection in oncology Transcriptomics: gene expression panel tests for cancer prognosis and treatment decisions } \\
\text { Pathogen genomics: tuberculosis whole genome sequencing } \\
\text { Whole genome sequencing (WGS) for outbreak management in hospitals } \\
\text { 3D imaging and printing: surgical guides, anatomical models, custom implants }\end{array}$ \\
\hline $\begin{array}{l}\text { Outcomes } \\
\text { Equalizing healthcare } \\
\text { Reducing mortality rate and human error } \\
\text { Reducing medical costs } \\
\text { Reducing reliance on social services }\end{array}$ \\
\hline
\end{tabular}

of drugs, both direct (i.e. medication prompts, 'befriending', AI art and virtual reality enironments to improve social isolation, loneliness, anxiety, depression, gait, posture and even pain in older adults) and indirect care (general maintenance of care environment, cleaning for example, development of optimised supply chains etc.) represent areas where AI technology can make a difference to the health and social care provision for the older person.

\section{Rise of Al in medicine}

The health system has accumulated massive datasets, largely due to the introduction of electronic records, which include demographic information, medical history, laboratory tests and radiological investigations, history of surgical interventions, medication history and allergies, lifestyle etc. Such data can be used productively for: improving diagnosis and treatment, prevention, diagnosis and cure of communicable, acute and chronic diseases, considering information on lifestyle, general health and demographics, aid timely diagnosis and prediction/prevention of disease onset at an early stage. A promising avenue is the secondary use of electronic health records, where patient data are analyzed to conduct clinical and translational research. One of the advantages of $\mathrm{AI}$ is its use in geographically isolated areas, where there is limited access to healthcare, and also overcoming the increasing lack of specialized medical staff. In this, machine learning (ML) and deep learning (DL) are paving the way, enabling extensive data sets to be analyzed using algorithms, a set of rules given to an AI program to find patterns which are far too complex or numerous for a human programmer to extract and teach a machine to recognize (Figure 1).
There are increasing examples of good AI practice in medicine. The Deep Patient project uses DL to analyze patient electronic health records to determine those most likely to develop serious illness within the next year. The AI algorithm was trained on 12 years of patient records $-700,000$ in all. When tested, it could predict risk for dozens of diseases including heart failure, several types of cancer, congestive heart failure and acute myocardial infarcts, as well as diabetes, schizophrenia and attention deficit and disruptive behavior disorders [5].

Accelerated DL techniques detect genetic mutations by

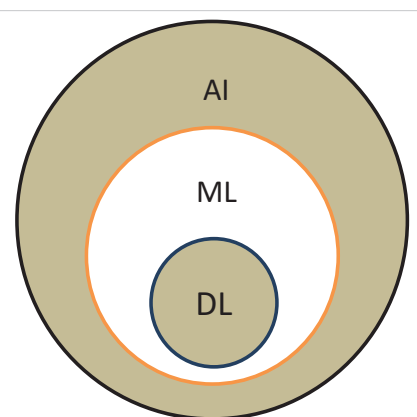

Figure 1: Understanding Al, machine learning and deep learning $A I$ is an umbrella term for machine learning $(M L)$ and deep learning $(D L)$, based on principles of machines completing tasks built on stipulated rules that solve problems using computer algorithms.

$\mathrm{ML}$ is a subset of Al where algorithms use statistical models to perform a specific task without explicit instructions to make informed decisions. Once predictive accuracy level is high enough, the machine 'learnt' the task.

A subset of $M L$, called $D L$ is based on brain-inspired systems, artificial neural networks (ANNs; types of algorithms that imitate how human neurons make decisions). They are excellent tools for finding patterns which are far too complex or numerous and require large data sets for training in order to deliver accurate results and be able to automatically discover features used for decision making for a particular (health) classification.

Abbreviations: Al: Artificial Intelligence; ML: Machine Learning; DL: Deep Learning. 
analyzing Magnetic Resonance Imaging (MRI) images in brain cancer with an accuracy between $63 \%$ - 69\% [6]. This method appears to be successful in identifying methylation of the 06methylguanine methyltransferase (MGMT) gene status and thus predicts response to treatment and prognosis in people with brain tumours. The accuracy increases (94.90\%) when using ResNet50 (50 layers) architecture [7]. This is further evidence that deep neural architectures can be used to predict molecular biomarkers from routine medical images and thereby avoid the need for brain biopsies. Such findings can be taken forward in therapeutic planning by predicting how patients with low-grade brain tumours will respond to chemo and radiotherapy.

\section{Examples of ML and DL implementation in the medical care of older people}

Both DLand MLare based on recognizing patterns of images and require thousands to millions data points to construct an AI learning model that will provide both an accurate and quick fit for use in medical purposes, i.e. computer-aided diagnosis. The conducted studies on older adults using ML and DL modelling to date use a small number of patients and lack reproducibility needed for wider clinical application in different clinical settings and larger populations. Furthermore, despite their high accuracy, their sensitivity and specificity for certain medical conditions, i.e. Alzheimer's disease (AD) and idiopathic Normal Pressure Hydrocephalus (iNPH), may differ, and even lag the sensitivity and specificity of a clinically trained professional [8].

The accuracy of AI algorithms is highest depending on numbers of variables, i.e. in identifying short-term adverse events after syncope in older people (95\% accuracy, based on ten variables from patient history, ECG, and the circumstances of syncope in a total number of 1844 subjects [9]). Increasing the number of variables $(n=27)$, even in a smaller sample [ $n$ $=49,18$ with documented history of traumatic brain injury (TBI) exposure and 31 without] results in higher accuracy of algorithms (98\%), linking autonomous nervous system with $\mathrm{TBI}$ and its progression to $\mathrm{AD}[10]$.

Higher accuracy of algorithms was also confirmed in a Finish study on a late-onset dementia prediction, based on 709 cognitively normal subjects at baseline who had a second re-examination up to 10 years later (incident dementia $n=39$ ). The algorithm was re-examined in an extended population ( $n=1009$, incident dementia 151). Accuracy of the developed model was $79 \%$ and $75 \%$ for the main and extended populations, with the main predictors for developing dementia being cognition, vascular factors, age, subjective memory complaints, and APOE genotype [11]. This algorithm can be useful for identifying individuals who are both at the highest risk of developing dementia and will benefit from dementia prevention interventions.

A similar method of developing an AI algorithm has also been used in identifying frailty and establishing the risk for falls in older people following an emergency department visit. Thus, the evaluation of several machine learning methodologies of automated risk stratification and referral intervention led to a predictive model with an accuracy of 78\% [12]. This is very similar to the accuracy of an AI algorithm used in identifying frailty among residents aged 75 years and over (75\%) [13]. Another study, based on demographic and psychometric data from 284 patients, aimed to predict the likelihood of older adults having persistent depressive symptoms after 12 months, using a ML approach. This approach provided a superior predictive performance using ML compared to logistic regression (mean accuracy $72 \%$ vs. 67\% $p<0.0001$ ) [14]. Similar accuracy (75\%) was also confirmed when using deep learning neural networks to model fall risk on the basis of accelerometer data to in particular multi-task learning, effectively assess fall risk on the basis of wearable sensor data [15]. It remains now to explore possibilities into translating these findings into a prevention programme and implement them in a routine clinical setting.

A most recent study used a novel residual extraction approach, based on anomaly detection technique where the image is compressed and reconstructed [8]. This novel DL method is particularly suited for small datasets and seems to be highly accurate in diagnosing dementia. Thus, [8] used a clinical set consisting of 69 subjects all in their 70s (23 in each group of iNPH, AD and control subjects) who underwent 3T MRI brain scans., and their whole-brain three-dimensional (3D) T1-weighted images were used to design a fully automated, 3D DL classifier to differentiate dementia from cognitively intact older people. They reported higher accuracy (90\%), with this method being more accurate than the neuroradiologically established diagnosis of $\mathrm{AD}$, but not iNPH.

In asimilarstudy, the DLbasedalgorithm, usingneuroimaging measures of Diffusion-Tensor Imaging (DTI) and MRI data [16] from 35 amyloid- $\beta 42$ negative mild cognitive impairment (MCI) subjects, 35 positive MCI subjects (amyloid- $\beta 42$ positive) and 25 healthy controls, DTI data had a better prediction accuracy than grey matter volume in preclinical AD dementia (77\% vs. 68\% accuracy respectively). Language in people with dementia has similarly been subjected to ML analysis and shown to discriminate well between people with AD and those with mixed (vascular and AD) dementia [17].

Besides the high accuracy in detecting dementia, an artificial neural network (ANN) algorithm, based on a large sample and datasets, has also been shown to identify risk factors for Alzheimer's disease [18]. Thus, age, lower education level and monthly family income may increase a person's risk for developing $\mathrm{AD}$, with a family history of dementia and physical inactivity also contributing to developing the disease. Interestingly, from known peripheral markers for dementia, it was urinary AD7c-NTP, but not blood $A \beta 42$, that was clinically valuable for the early diagnosis of $\mathrm{AD}$ [18]. The final 
established ANN containing multiple information including epidemiological parameters, neuropsychological functions and biomarkers obtained a high diagnostic precision and efficiency, and it has a potential to be used as an effective lowcost tool for screening and diagnosis of dementia in general and dementia subtypes.

ML is more accurate in respect to statistical methods to predict acute kidney injury in older people [19] and hospitalacquired pneumonia in people with high risk, i.e. mentally ill people, treated with neuroleptic medication [20], functional fall risk [21] and other poor outcomes in older adults, including delirium [22] and the overall risk of emergency admission [23]. The described algorithms have a potential to provide a more complete and accurate assessment of effects of ageing and risk for physical illnesses and falls in older people while providing a clinically useful predictive capability for earlier intervention in those patients at greatest risk of developing them.

Another useful contribution of AI is the development of novel pharmacological interventions based on virtual screening and de novo drug design using DL [24]. Novel pharmacological discoveries will hopefully avoid the adverse effects of current therapies used in older people, while reducing costs and risks related to preclinical and clinical trials. In addition, DL models can also help identify putative preventive measures (i.e. family support, socioeconomic status and friendship activity) in managing cerebrovascular disease, hearing loss and cognitive impairment in middle-aged and older adults [25].

These technological advances, in return, have the potential to contribute towards updating social policy needs and improve the socioeconomic status and health conditions of older adults.

\section{Assisted technology in geriatric care and rehabilitation: Robots and telemedicine}

Maintaining mobility is fundamental to independent living and quality of life of older people. Robotic and sensor technologies could make a significant difference in the lives of older people and their caregivers. A recent review of 36 studies on sensor technologies and the use of robots for supporting older people with mobility limitations highlighted the potential for their use even without the physical presence of medical personnel [26]. Robotic exercise appears to be more effective in improving dynamic balance and lower extremity muscle strength than conventional exercise in frail people [27]. The Robot Gym, an alternative to traditional physical and occupational therapy approaches in post-stroke rehabilitation, enhanced stroke patients' functionality both in upper and lower extremity tests and was more successful in improving lower limb function than traditional therapy [28]. In another study, participants with chronic hemiparetic gait improved on treadmill-integrated training but not seated ankle robotics training [29]. Another use of robots in rehabilitation is dancing, used to improve balance and gait in people with Parkinson's disease and older adults. A wheeled mobile robot with a human-like upper-body was shown to be a good dance follower, with $50 \%-60 \%$ of expert dancers agreeing in its efficacy [30]. These studies suggest Robot Gyms can be as effective as traditional therapy for people with mobility problems, presenting cost and labour-efficient options for countries with scarce clinical resources and funding.

Artificial conversationalists, chatbots communicating via auditory or textual methods, are another method of care delivery that reduces people's loneliness by providing social support. A survey of older people's needs highlighted several uses of robotics embedded in the environment (i.e. furniture, walls, ceiling, etc.) to offer enhanced support and maintain activities of daily living [31]. Voice-controlled intelligent personal assistants (i.e. Amazon Echo and Google Home) similarly can provide companionship, reminders, emergency communication and even entertainment for older people living alone, while also reducing caregivers' burden [32]. However, these services are in early-stage development and need to be both standardized and properly validated for largescale industrial manufacture.

Interactions with robots affect people's blood pressure and psychosocial wellbeing. Thus, systolic and diastolic blood pressure decreased significantly from baseline in residents following an interaction with a seal companion robot, Paro [33]. Similarly, reported loneliness and depression decreased due to discussing and talking to the robot [34]. Robopets in general, including Paro, have a positive impact on the general physical health, well-being and quality of life among older people living in care homes, and also significantly reduce agitation (by 68\%), as shown in a systematic review of 19 studies [35]. Nutritional aspects of care also benefit from robot interactions [36].

Telecare systems are another platform in remotely assisting frail people. A robot named ROBIN is integrated into a sensorrich environment and continuously monitors the physical and psychological wellbeing of older persons living alone, with caregivers communicating through it to their assisted persons. This enhanced telepresence robot, as assessed by users for its suitability for supporting social interaction and providing motivational feedback on health-related aspects, was judged to be usable and interactively pleasing [37]. It is thus not surprising that a robot-administered cognitive testing has been well accepted by community-dwelling older adults [38]. Contrary to expectations, older people seem to accept and respond well to the use of these novel enabling technologies for remote assistance, social communication and medical care, highlighting the importance of incorporating their needs and preferences in developing effective technological solutions. In addition, when faced with a choice, they seem to prefer a service-oriented robot over the more companion-oriented robot [39]. 
Table 2: Ethical issues regarding Al use in medicine.

\begin{tabular}{|c|c|}
\hline $\begin{array}{l}\text { Service users and } \mathrm{Al} \\
\text { interphase }\end{array}$ & $\begin{array}{l}\text { Do patents have the right to refuse diagnostics and treatment planning by Al? } \\
\text { Are patients being offered a justification for holding their health data for use in algorithmic decisions? } \\
\text { What are the ethical issues about virtual reality treatment in vulnerable patients, i.e. those with dementia and learning disability? } \\
\text { How will the relationship between physicians and patients change as a result of the introduction of algorithms in the provision of medical care? }\end{array}$ \\
\hline $\begin{array}{c}\text { Technical and } \\
\text { human interphase }\end{array}$ & $\begin{array}{c}\text { How to spot and correct errors contained in Al algorithms and prevent unforeseen consequences and unfair outcomes along economic and racial } \\
\text { class lines? This is an important issue since the quality of decision-making is based on quality assurance of data, and thus has direct implications for } \\
\text { clinical decisions and communicating them to patients [40]; } \\
\text { Who is going to attend to (un)identified methodological shortcomings? } \\
\text { Lack of IT and Al educated medical teams to manage Al effectively (physicians must adequately understand how algorithms are created, critically } \\
\text { assess the source of the data used to create statistical models designed to predict outcomes, facilitate diagnosis or treatments, understand how } \\
\text { models function and guard against becoming overly dependent on them); } \\
\text { What about monitoring technological developments to ensure preventative and precautionary safeguards for those who are involved? } \\
\text { Technological crime? }\end{array}$ \\
\hline $\begin{array}{l}\text { Moral and Al } \\
\text { interphase }\end{array}$ & $\begin{array}{l}\text { What about building moral robots? This will require educating researchers and engineers about moral values and ethics; } \\
\text { Algorithms can be designed to perform in unethical ways: dismissing or failure to notice those who lack access to care; under testing those who } \\
\text { cannot pay; race or gender biases; algorithms may introduce problematic decisions that reflect biases inherent in data used to train them [41] } \\
\text { algorithms might be designed to skew results, depending on motives of programmers, companies or health care systems; } \\
\text { Data gathered about patient health, diagnostics and outcomes may be used without regard for clinical experience and the human aspect of patient care; } \\
\text { Tension between goals of improving health and generating profit. }\end{array}$ \\
\hline
\end{tabular}

\section{Concerns about Al use in medicine}

Despite developers' stated confidence in ML and DL to accurately predict diagnosis and outcomes of medical illnesses, the evidence for their successful implementation in clinical care remains scarce [40], with several concerns being raised: ethical issues of privacy by healthcare service users, professionals sand stakeholders [41,42], equity, security [40], quality of data, lack of data re patients outcomes and cost of care [43], data management and human contact [42]. Divergences emerged in relation to how these ethical issues are interpreted, how conflicts between different ethical principles are resolved and what solutions should be implemented to overcome current challenges (Table 2). The Canadian Association of Radiologists recently provided a framework for the study of legal and ethical issues related to $\mathrm{AI}$ in medical imaging for patient data (privacy, confidentiality, ownership, and sharing), algorithms (levels of autonomy, liability and jurisprudence), practice (best practices and current legal framework) and opportunities from the perspective of a universal healthcare system [44-46]. Though the authors acknowledged the benefits ML can have on patients' health, they also caution that full benefit of AI to make predictions and take alternative actions should also consider the accompanying ethical pitfalls. Not surprisingly, the most recent report by the USA National Academy of Medicine prioritized ethical issues in the delivery of personalized AI health care calling for regulation and legislation of AI medical innovations [43].

\section{Will Al change the face of modern geriatric medicine?}

There is always a need for continuous supervision and quick diagnosis in the case of older patients. The promise of modernizing the health system by delivering an efficient, precise, person-centred and cost-effective healthcare has been a driver to implement new technologies. However, very little has been delivered to date in terms of direct patients' care and benefits. The ethical issues remain unaddressed and without this regulated the wider AI implementation within the healthcare system cannot proceed. Health professionals should not blindly embrace technological advances but instead take them carefully when discussing algorithm-driven clinical decisions, include ML in multidisciplinary meetings, while additionally learningnewskillsin statistics and computer science to help develop the clinical algorithms and their evaluation in routine clinical practice. This will require evolving the general healthcare culture, updating current medical curricula and training future doctors with new diagnostic and management concepts. In addition, acceptance of digital clinical decisions should be approved by healthcare regulatory organizations, so that legal and clinical backing is required, similar to various NICE guidelines. Considering the novel and potential impact of AI on future healthcare systems, consideration must be given to legal, ethical and social implementation with all stakeholders before its implementation including patients, public, and a wide range of healthcare providers to enable its meaningful development.

\section{References}

1. Jiang $F$, Jiang $Y$, Zhi H, Dong $Y$, Li H, et al. Artificial intelligence in healthcare: past, present and future. Stroke Vasc Neurol. 2017; 2: 230-243. PubMed: https://www.ncbi.nlm.nih.gov/pubmed/29507784

2. Hargreaves DS, Sizmur S, Viner RM. Do young and older adults have different health care priorities? Evidence from a national survey of English inpatients. J Adolesc Health. 2012; 51: 528-532.

PubMed: https://www.ncbi.nlm.nih.gov/pubmed/23084177

3. Lafortune C, Huson K, Santi S, Stolee P. Community-based primary health care for older adults: a qualitative study of the perceptions of clients, caregivers and health care providers. BMC Geriatr. 2015; 15: 57.

4. Age UK. Briefing: Health and Care of Older People in England 2017. 2017. https://www.ageuk.org.uk/Documents/EN-GB/For-professionals/ Research/The_Health_and_Care_of_Older_People_in_England_2016. pdf?dtrk=true

5. Miotto R., Li L, Kidd BA, Dudley JT. Deep patient: An unsupervised representation to predict the future of patients from the electronic health records. Sci Rep. 2016; 6: 26094.

6. Kickingereder P, Bonekamp D, Nowosielski M, Kratz A, Sill M, et al Radiogenomics of glioblastoma: Machine learning-based classification of molecular characteristics by using multiparametric and multiregional MR imaging features. Radiology. 2016; 281: 907-918. PubMed: https://www.ncbi.nlm.nih.gov/pubmed/27636026 
7. Korfiatis P, Kline TL, Lachance DH, Parney IF, Buckner JC, et al. Residual deep convolutional neural network predicts MGMT methylation status. J Digit Imaging. 2017; 30: 622-628.

PubMed: https://www.ncbi.nlm.nih.gov/pubmed/28785873

8. Irie R, Otsuka $\mathrm{Y}$, Hagiwara A, Kamagata K, Kamiya K, et al. A Novel Deep Learning Approach with a 3D Convolutional Ladder Network for Differential Diagnosis of Idiopathic Normal Pressure Hydrocephalus and Alzheimer's Disease. Magn Reson Med Sci. 2020.

PubMed: https://www.ncbi.nlm.nih.gov/pubmed/31969525

9. Costantino G, Falavigna G, Solbiati M, Casagranda I, Sun BC, et al Neural networks as a tool to predict syncope risk in the Emergency Department Europace. 2017; 19: 1891-1895

PubMed: https://www.ncbi.nlm.nih.gov/pubmed/28017935

10. Ho L, Legere M, Li T, Levine $\mathrm{S}$, Hao K, et al. Autonomic nervous system dysfunctions as a basis for a predictive model of risk of neurological disorders in subjects with prior history of traumatic brain injury: Implications in Alzheimer's Disease. J Alzheimers Dis. 2017; 56: 305-315. PubMed: https://www.ncbi.nlm.nih.gov/pubmed/27911325

11. Pekkala T, Hall A, Lötjönen J, Mattila J, Soininen H, et al. Development of a late-life Dementia Prediction Index with supervised machine learning in the population-based CAIDE Study. J Alzheimers Dis. 2017; 55: 1055-1067.

PubMed: https://www.ncbi.nlm.nih.gov/pubmed/27802228

12. Patterson BW, Engstrom CJ, Sah V, Smith MA, Mendonça EA, et al. Training and Interpreting Machine Learning Algorithms to Evaluate Fall Risk After Emergency Department Visits. Med Care. 2019; 57: 560-566. PubMed: https://www.ncbi.nlm.nih.gov/pubmed/31157707

13. Ambagtsheer RC, Shafiabady N, Dent E, Seiboth C, Beilby J. The application of artificial intelligence (Al) techniques to identify frailty within a residential aged care administrative data set. Int J Med Inform. 2020; 136: 104094.

PubMed: https://www.ncbi.nlm.nih.gov/pubmed/32058264

14. Hatton CM, Paton LW, McMillan D, Cussens J, Gilbody S, et al Predicting persistent depressive symptoms in older adults: A machine learning approach to personalised mental healthcare. J Affect Disord. 2019; 246: 857-860.

PubMed: https://www.ncbi.nlm.nih.gov/pubmed/30795491

15. Nait Aicha A, Englebienne G, van Schooten KS, Pijnappels M, Kröse B. Deep Learning to Predict Falls in Older Adults Based on Daily-Life Trunk Accelerometry. Sensors (Basel). 2018; 18: 1654.

PubMed: https://www.ncbi.nlm.nih.gov/pubmed/29786659

16. Dyrba M, Barkhof F, Fellgiebel A, Filippi M, Hausner L, et al. Predicting prodromal Alzheimer's Disease in subjects with Mild Cognitive Impairment using machine learning classification of multimodal multicentre Diffusion-Tensor and Magnetic Resonance Imaging data. J Neuroimaging. 2015; 25: 738-747.

PubMed: https://www.ncbi.nlm.nih.gov/pubmed/25644739

17. Rentoumi V, Raoufian L, Ahmed S, de Jager CA, Garrard P. Features and machine learning classification of connected speech samples from patients with autopsy proven Alzheimer's disease with and without additional vascular pathology. J Alzheimers Dis. 2014; 42. PubMed: https://www.ncbi.nlm.nih.gov/pubmed/25061045

18. Wang $\mathrm{N}$, Chen $\mathrm{J}$, Xiao $\mathrm{H}, \mathrm{Wu} \mathrm{L}$, Jiang $\mathrm{H}$, et al. Application of artificial neural network model in diagnosis of Alzheimer's disease. BMC Neurol. 2019; 19: 154.

PubMed: https://www.ncbi.nlm.nih.gov/pubmed/31286894

19. Davis SE, Lasko TA, Chen G, Siew ED, Matheny ME. Calibration drift in regression and machine learning models for acute kidney injury. J Am Med Inform Assoc. 2017; 24: 1052-1061.

PubMed: https://www.ncbi.nlm.nih.gov/pubmed/28379439
20. Kuo KM, Talley PC, Huang $\mathrm{CH}$, Cheng LC. Predicting hospital-acquired pneumonia among schizophrenic patients: a machine learning approach. BMC Med Inform Decis Mak. 2019; 19: 42. PubMed: https://www.ncbi.nlm.nih.gov/pubmed/30866913

21. Iluz T, Weiss A, Gazit E, Tankus A, Brozgol M, et al. Can a Body-Fixed Sensor Reduce Heisenberg's Uncertainty When It Comes to the Evaluation of Mobility? Effects of Aging and Fall Risk on Transitions in Daily Living. J Gerontol A Biol Sci Med Sci. 2016; 71: 1459-1465. PubMed: https://www.ncbi.nlm.nih.gov/pubmed/25934996

22. Corradi JP, Thompson S, Mather JF, Waszynski CM, Dicks RS Prediction of Incident Delirium Using a Random Forest classifier. J Med Syst. 2018; 42: 261.

PubMed: https://www.ncbi.nlm.nih.gov/pubmed/30430256

23. Rahimian F, Salimi-Khorshidi G, Payberah AH, Tran J, Ayala Solares $\mathrm{R}$, et al. Predicting the risk of emergency admission with machine learning: Development and validation using linked electronic health records. PLoS Med. 2018; 15: e1002695.

PubMed: https://www.ncbi.nlm.nih.gov/pubmed/30458006

24. Zhong F, Xing J, Li X, Liu X, Fu Z, et al. Artificial intelligence in drug design. Sci China Life Sci. 2018; 61: 1191-1204.

PubMed: https://www.ncbi.nlm.nih.gov/pubmed/30054833

25. Lee KS, Park KW. Social determinants of the association among cerebrovascular disease, hearing loss and cognitive impairment in a middle-aged or older population: Recurrent neural network analysis of the Korean Longitudinal Study of Aging (2014-2016). Geriatr Gerontol Int. 2019; 19: 711-716.

PubMed: https://www.ncbi.nlm.nih.gov/pubmed/31257714

26. Penteridis L, D'Onofrio G, Sancarlo D, Giuliani F, Ricciardi F, et al. Robotic and sensor technologies for mobility in older people. Rejuvenation Res. 2017; 20: 401-410.

PubMed: https://www.ncbi.nlm.nih.gov/pubmed/28482748

27. Ozaki K, Kondo I, Hirano S, Kagaya H, Saitoh E, et al. Training with a balance exercise assist robot is more effective than conventional training for frail older adults. Geriatr Gerontol Int. 2017; 17: 1982-1990. PubMed: https://www.ncbi.nlm.nih.gov/pubmed/28295912

28. Bustamante Valles K, Montes S, Madrigal Mde J, Burciaga A, Martínez $M E$, et al. Technology-assisted stroke rehabilitation in Mexico: a pilot randomized trial comparing traditional therapy to circuit training in a Robot/technology-assisted therapy gym. J Neuroeng Rehabil. 2016; 13: 83.

PubMed: https://www.ncbi.nlm.nih.gov/pubmed/27634471

29. Forrester LW, Roy A, Hafer-Macko C, Krebs HI, Macko RF. Task-specific ankle robotics gait training after stroke: a randomized pilot study. $J$ Neuroeng Rehabil. 2016; 13: 51.

PubMed: https://www.ncbi.nlm.nih.gov/pubmed/27255156

30. Chen TL, Bhattacharjee T, McKay JL, Borinski JE, Hackney ME, et al Evaluation by Expert Dancers of a Robot That Performs Partnered Stepping via Haptic Interaction. PLoS One. 2015; 10: e0125179. PubMed: https://www.ncbi.nlm.nih.gov/pmc/articles/PMC4438977/

31. Güttler J, Georgoulas C, Linner T, Bock T. Towards a future robotic home environment: A survey. Gerontology. 2015; 61: 268-80. PubMed: https://www.ncbi.nlm.nih.gov/pubmed/25341658

32. O'Brien K, Liggett A, Ramirez-Zohfeld V, Sunkara P, Lindquist LA. VoiceControlled Intelligent Personal Assistants to Support Aging in Place. J Am Geriatr Soc. 2020; 68: 176-179.

PubMed: https://www.ncbi.nlm.nih.gov/pubmed/31617581

33. Robinson H, MacDonald B, Broadbent E. Physiological effects of a companion robot $\mathrm{n}$ blood pressure of older people in residential care facility: a pilot study. Australas J Ageing. 2015; 34: 27-32. PubMed: https://www.ncbi.nlm.nih.gov/pubmed/24373064 
34. Abbott R, Orr N, McGill P, Whear R, Bethel A, et al. How do "robopets" impact the health and well-being of residents in care homes? A systematic review of qualitative and quantitative evidence. Int $\mathrm{J}$ Older People Nurs. 2019; 14: e12239.

PubMed: https://www.ncbi.nlm.nih.gov/pubmed/31070870

35. Robinson H, Macdonald B, Kerse N, Broadbent E. The psychosocia effects of a companion robot: a randomized controlled trial. J Am Med Dir Assoc. 2013; 14: 661-667.

PubMed: https://www.ncbi.nlm.nih.gov/pubmed/23545466

36. Łukasik S, Tobis S, Wieczorowska-Tobis K, Suwalska A. Could Robots Help Older People with Age-Related Nutritional Problems? Opinions of Potential Users. Int J Environ Res Public Health. 2018; 15.

PubMed: https://www.ncbi.nlm.nih.gov/pubmed/30424561

37. Cortellessa G, Fracasso F, Sorrentino A, Orlandini A, Bernardi G, et al. ROBIN, a telepresence robot to support older users monitoring and social inclusion: Development and evaluation. Telemed J E Health. 2018; 24: 145-154.

PubMed: https://www.ncbi.nlm.nih.gov/pubmed/28771398

38. Takaeda K, Kamimura T, Inoue T, Nishiura Y. Reliability and acceptability of using a social robot to carry out cognitive tests for communitydwelling older adults. Geriatr Gerontol Int. 2019; 19: 552-556. PubMed: https://www.ncbi.nlm.nih.gov/pubmed/30884153

39. Chu L, Chen HW, Cheng PY, Ho P, Weng IT, et al. Identifying Features that Enhance Older Adults' Acceptance of Robots: A Mixed Methods Study. Gerontology. 2019; 65: 441-450.

PubMed: https://www.ncbi.nlm.nih.gov/pubmed/30844813
40. Car J, Sheikh A, Wicks P, Williams MS. Beyond the hype of big data and artificial intelligence: Building foundations for knowledge and wisdom. BMC Med. 2019; 17: 143.

41. Wang S, Bolling K, Mao W, Reichstadt J, Jeste $D$, et al. Technology to Support Aging in Place: Older Adults' Perspectives. Healthcare (Basel). 2019; 7.

PubMed: https://www.ncbi.nlm.nih.gov/pubmed/30974780

42. Wangmo $T$, Lipps $M$, Kressig RW, lenca M. Ethical concerns with the use of intelligent assistive technology: findings from a qualitative study with professional stakeholders. BMC Med Ethics. 2019; 20: 98. PubMed: https://www.ncbi.nlm.nih.gov/pubmed/31856798

43. Matheny ME, Whicher D, Thadaney Israni S. Artificial Intelligence in health Care: A report from the National Academy of Medicine. JAMA. 2019.

PubMed: https://www.ncbi.nlm.nih.gov/pubmed/31845963

44. Miller DD. The medical Al insurgency: what physicians must know about data to practice with intelligent machines. NPJ Digit Med. 2019; 2: 62.

45. Char DS, Shah NH, Magnus D. Implementing machine learning in health care - addressing ethical challenges. N Engl J Med. 2018; 378: 981-983. PubMed: https://www.ncbi.nlm.nih.gov/pubmed/29539284

46. Jaremko JL, Azar M, Bromwich R, Lum A, Alicia Cheong LH, et al. Canadian Association of Radiologists (CAR) Artificial Intelligence Working Group. Canadian Association of Radiologists White Paper on ethical and legal issues related to Artificial Intelligence in radiology. Can Assoc Radiol J. 2019; 70: 107-118.

PubMed: https://www.ncbi.nlm.nih.gov/pubmed/30962048 